\title{
Novel Disposable Microelectrode Array for Cultured Neuronal Network Recording Exhibiting Equivalent Performance to Commercially Available Arrays
}

Hamid Charkhkar ${ }^{1}$, David E. Arreaga-Salas ${ }^{2}$, Thao Tran ${ }^{3}$, Audrey Hammack ${ }^{4}$, Walter E. Voit ${ }^{2,5}$, Joseph J. Pancrazio ${ }^{3,5}$ Bruce E. Gnade ${ }^{2}$

${ }^{1}$ George Mason University, Department of Electrical and Computer Engineering, 4400 University Drive, 1G5, Fairfax, VA 22030 USA.

${ }^{2}$ University of Texas at Dallas, Department of Materials Science \& Engineering, Richardson, TX 75080 USA.

${ }^{3}$ George Mason University, Department of Bioengineering, 4400 University Drive, 1G5, Fairfax, VA 22030 USA

${ }^{4}$ University of Texas at Dallas, Department of Chemistry, Richardson, TX 75080 USA.

${ }^{5}$ University of Texas at Dallas, Department of Bioengineering, Richardson, TX 75080 USA.

Corresponding author:

Hamid Charkhkar, $\mathrm{PhD}$

Present Address:

Advanced Platform Technology (APT) Center

Louis Stokes Cleveland VA Medical Center

10701 East Blvd (151 W), Cleveland, OH 44106, USA

Email: hamid.charkhkar@case.edu;

Tel: +1 216-791-3800 ext. 2924

Fax: +1 216-707-6420 


\section{Abstract}

2 Microelectrode arrays (MEAs) enable non-invasive recording of supra-threshold signals, i.e.

3 action potentials or spikes, from a variety of cultured electrically active cells. While this label-

4 free technology has been shown to be useful for pharmacological and toxicological studies, a

5 major limitation has been the reliance on expensive recording substrates that have been

6 manufactured with the intent of re-use. Prior work by our group has demonstrated an approach

7 for fabricating MEAs using conventional liquid crystal display manufacturing techniques. Here,

8 we describe and characterize the UT Dallas planar MEA which is fabricated with low cost

9 materials and processes. We compare the performance of the UT Dallas MEAs, which consist of 10 exposed gold microelectrodes with patterned parylene insulation over traces, with well-

11 established commercially available MEAs using cultured murine cortical networks. Detailed

12 electrophysiological comparisons show virtually identical performance between MEA types with

13 respect to network metrics including recording yield across the array, network spike rate and

14 burst rate, and virtually identical pharmacological responses to a diverse set of 15 neuropharmacological agents.

17 Keywords: Microelectrode array, Disposable microelectrodes, Extracellular recording, in vitro 18 assay, Neuronal recording

\section{Introduction}

Neuronal networks cultured on planar microelectrode arrays (MEAs) have utility in wide

22 range of in vitro applications including neuropharmacology [1-4], assessing biocompatibility of

23 novel materials [5], studying neural network plasticity [6-8], connectivity [9], and response to

24 electrical stimulation [10,11], as well as the emergence of dynamic states [12,13]. Murine 
1 primary cultures derived from embryonic neural tissue mature to form spontaneously active

2 networks on the surface of MEAs. . In fact, the use of cultured cortical tissue on MEAs for

3 pharmacological assays has been cross-validated across multiple laboratories [14]. Compared to

4 other electrophysiological assay or imaging approaches, MEAs offer label-free, non-invasive,

5 and long-term recording capabilities.

6 To create MEAs with features on the order of tens of microns, fabrication processes and

7 materials common in the silicon microelectronics industry are used. Common conductive

8 materials comprising MEAs include indium tin oxide (ITO), platinum, gold, or titanium nitride,

9 whereas the insulating materials include SU-8, silicon nitride, polymimide, parylene, or

10 polydimethylsiloxane. Advances in MEA technology have mainly focused on increasing spatial

11 resolution and channel density through the incorporation of on-chip CMOS electronics [15-18].

12 Unfortunately, MEAs are typically expensive, fragile, and consist of materials that are distinctly

13 different from conventional polystyrene culture dishes used and discarded in typical cell biology

14 laboratories. Manufacturers expect re-use and specialized handling of these devices.

We previously reported on the application of liquid crystal display fabrication

16 technologies to create a low-cost functional MEA [19] that made use of gold microelectrode

17 contacts and traces insulated with parylene-C, a biocompatible polymer often used in 18 implantable device applications [20-22].

In the present paper, we demonstrate reproducible fabrication of low-cost MEAs

20 leveraging our fabrication process that exhibit stable electrochemical impedance profiles of gold

21 contacts and conducting polymer modified sites under culture conditions. Developed at the

22 University of Texas at Dallas (UT Dallas), we show that the performance of these MEAs with

23 respect to cultured neuronal network recording is entirely consistent with that from well- 
1 established commercially available MEAs from Multi Channel Systems (Reutlingen, Germany).

2 Our data show that the UT Dallas MEAs offer a platform for low-cost, disposable high content 3 assays from neuronal networks.

4

5 2. Methods

$6 \quad 2.1$ Fabrication and characterization

An array of 60 gold square microelectrodes $30 \mu \mathrm{m}$ in length was patterned on the

8 substrate with insulation over the leads with parylene-C. The outer dimensions of the array

9 substrate were $3.8 \mathrm{~cm} \times 3.8 \mathrm{~cm}$ with $2 \mathrm{~mm} \times 2 \mathrm{~mm}$ bond pads separated by $400 \mu \mathrm{m}$. As shown in

10 Fig.1, the substrate was a $500 \mu \mathrm{m}$ thick layer of polycarbonate, a stiffer material than

11 polyethylene naphthalate used previously [19], which improves ease of handling. The gold

12 microelectrodes were deposited by e-beam evaporation and patterned using standard

13 photolithography and wet etch. Parylene-C was deposited by chemical vapor deposition and then

14 exposed to oxygen reactive ion etching for patterning.

15 Electrochemical characterization of the MEA was performed by electrochemical 16 impedance spectroscopy (EIS) on individual microelectrodes from a typical array. The 17 measurements were performed using a two-electrode setup using a potentiostat/galvanostat $(\mathrm{CH}$ 18 600D, CH Instruments, Texas, US) equipped with an electrochemical analyzer module (CHI 19 Version 9.03, CH Instruments). Measurements were made in the presence of phosphate-buffered 20 saline (PBS) at $\mathrm{pH}$ of 7.4 at room temperature by applying a sinusoidal signal with $20 \mathrm{mV}$ 21 amplitude over a range of frequencies from $10 \mathrm{~Hz}$ to $100 \mathrm{kHz}$ to characterize the complex 22 impedance of the working electrode and the electrolyte solution.

$24 \quad 2.2$ MEA preparation and cell culture 

model MCSMEA-S2-GR which consist of 60 ITO microelectrodes, were used for comparison with the novel MEAs. Microelectrodes had a diameter of 10 - $30 \mu \mathrm{m}$ and inter-electrode spacing of $200 \mu \mathrm{m}$. As described in [5], MCS MEAs were first disinfected by $70 \%$ ethanol for 20 minutes under laminar flow in a biohood and then rinsed with sterile de-ionized water. To obtain

6 better cell adhesion, the center regions of the MCS MEAs were coated with $50 \mu \mathrm{g} / \mathrm{ml}$ of poly-D-

7 lysine (PDL) (Sigma-Aldrich, St. Louis, MO) overnight. After the incubation with PDL, the

8 arrays were then washed with sterile deionized water three times to remove any excess PDL.

9 The arrays were then coated at the center with $20 \mu \mathrm{g} / \mathrm{mL}$ of laminin (Sigma-Aldrich, St. Louis, MO) for at least an hour. Prior to the cell seeding the laminin was removed from the MCS 11 MEAs.

Small, yet important modifications to the above preparation steps enabled immediate and 13 reliable use of the UT Dallas MEAs for cell culture. First, UT Dallas MEAs were exposed to 14 oxygen plasma treatment with the oxygen pressure of 15 psi for 1 min at $75 \mathrm{~W}$ (PE-50, Plasma Etch Inc, Carson City, NV). UT Dallas MEA wells were filled with PBS and allowed to soak for 1624 hours at room temperature. Under a biological containment hood, UT Dallas MEA wells were 17 treated with $70 \%$ ethanol for $20 \mathrm{~min}$, washed with sterile water, filled with cell culture medium 18 consisting of DMEM (Life Technologies), 2\% B27 (Life Technologies), 5\% horse serum 19 (Atlanta Biologicals, Lawrenceville, GA), 5\% fetal bovine serum (Life Technologies), and 0.2\% $204 \mathrm{mg} / \mathrm{ml}$ ascorbic acid (Sigma Aldrich) and incubated overnight at $37{ }^{\circ} \mathrm{C}$ in the cell culture 21 incubator. After removal of the culture medium, $\sim 50 \mu \mathrm{l}$ of $50 \mu \mathrm{g} / \mathrm{ml}$ of PDL was applied to the 22 center of each UT Dallas MEA recording well and allowed to incubate at $37^{\circ} \mathrm{C}$ in a $\mathrm{CO}_{2}$ 23 incubator overnight, with care taken to avoid evaporation. Approximately 2-3 hr prior to cell 
1 plating, PDL was washed from the recording wells three times with sterile water and the surface

2 was allowed to dry. Finally, $\sim 50 \mu \mathrm{l}$ of $20 \mu \mathrm{g} / \mathrm{ml}$ of laminin was applied for at least $1 \mathrm{hr}$ with the excess removed immediately prior to plating. Pilot experiments revealed that strict adherence to

4 the above preparatory steps enabled reliable adhesion of dissociated primary embryonic cortical 5 tissue and subsequent maturation into active networks.

The primary neuronal culture method was similar to that described in [23]. The

7 procedure was approved by the Institutional Animal Care and Use Committee of George Mason

8 University (Fairfax, VA). Timed pregnant, embryonic day 17, CD-1 mice (Charles River, 9 Wilmington, MA) were euthanized with carbon dioxide followed by decapitation. Embryos were extracted in ice cold L15 (Life Technologies, Grand Island, NY). Upon isolation of the 11 frontal cortex, the tissue was stored up to 24 hours in a hibernate media (BrainBits, Springfield, 12 IL) supplemented with 2\% B27 (Life Technologies) and 0.5 mM Glutamax (Life Technologies), 13 Later, the hibernate media was removed, the tissue was minced by scalpels and then dissociated 14 through incubation with DNAase and papain (Worthington Biochemical Corp., Lakewood, NJ) 15 for 15 minutes followed by mechanical trituration using disposable graduated pipettes (Fisher 16 Scientific, Pittsburg, PA). After centrifuging at $2500 \mathrm{rpm}$ for 5 minutes, the supernatant was 17 removed and cells were re-suspended in culture medium. The cells were counted using a 18 hemocytometer (Life Technologies) and immediately seeded on MEAs at a density of 100,000 in 19 a $50 \mu \mathrm{L}$ droplet. All cultures were incubated at $37^{\circ} \mathrm{C}$ with $10 \% \mathrm{CO}_{2}$ and maintained in DMEM 20 with GlutaMAX supplemented with 5\% horse serum (Atlanta Biologicals), 5\% FBS (Life 21 Technologies), 2\% B27, and $0.2 \%$ of $4 \mathrm{mg} / \mathrm{ml}$ ascorbic acid (Sigma-Aldrich) for the first two 22 days. To avoid overgrowth of glial cells, the fetal bovine serum was removed at day 3 and the 23 cultures were thereafter maintained by a 50\% media exchange twice a week for at least 21 days. 
2.3 Extracellular recordings and analysis

All recordings were performed after at least 3 weeks in vitro to ensure that the neural

4 networks had reached maturity and consistency in activity. The multichannel extracellular

5 recordings were acquired with an OmniPlex data acquisition system (Plexon Inc, Dallas, TX),

6 where each of the 60 channels of either MEA was digitized at a sampling frequency of $40 \mathrm{kHz}$.

7 During the recording session, the culture temperature was controlled at $37^{\circ} \mathrm{C}$.

8 During extracellular recordings from the neuronal networks, the mean noise level was

9 calculated for each individual channel and a threshold was set at 5 standard deviations from this

10 mean. A spike was then detected if the signal surpassed this threshold. Channels were

11 considered to be active if the spike rate was at least $0.1 \mathrm{~Hz}$. The recorded spikes from each

12 channel were sorted off-line into well-resolved units using Offline Sorter V.3 (Plexon Inc.). The

13 spike sorting method was based on the 2D principle component analysis of spike waveforms

14 followed by scanning K-Means to find and separate between clusters. Each unit presumably

15 corresponds to the signal from an individual neuron. Consistent with [24], a burst was defined as

16 the occurrence of a minimum of 4 spikes which were not more than $75 \mathrm{~ms}$ apart from each other.

17 The minimum inter-burst interval was set to $100 \mathrm{~ms}$.

\subsection{Pharmacological exposure}

20 For a subset of experiments, the pharmacological sensitivity of the cultured neuronal

21 networks on the MCS and novel MEAs was examined. In separate comparison experiments, we

22 applied the following compounds to the cultures on both MEA platforms: $20 \mu \mathrm{M}$ memantine

23 (Sigma -Aldrich), a blocker of excitatory N-methyl-D-aspartic acid (NMDA) receptors, $1 \mathrm{mM}$ 
1 pilocarpine (Sigma -Aldrich), a muscarinic cholinergic receptor agonist, $5 \mu \mathrm{M}$ amyloid beta

2 (A $\left.\beta_{1-42}\right)$ monomer (Anaspec, Fremont, $\mathrm{CA}$ ), a biomolecule associated with Alzheimer's disease, 3 and $5 \mu \mathrm{M}$ bicuculline, a blocker of $\mathrm{GABA}_{\mathrm{A}}$ receptors. The comparative effects of all the 4 compounds were examined under acute conditions.

\section{3. Results and discussion}

3.1 Microelectrode impedance stability

As shown in Fig. 2A, the measured impedance for representative electrodes paralleled previous observations for metal electrodes in saline solution [25]. To assess stability of the novel MEA microelectrodes and surrounding insulation, we compared the initial impedance and final impedance after 30 days within a cell incubator at $37{ }^{\circ} \mathrm{C}$. The initial impedance magnitude at 1

$12 \mathrm{kHz}$ was $796.3 \pm 49.6 \mathrm{k} \Omega$ (mean $\pm \mathrm{SEM}, \mathrm{n}=$ total of 16 microelectrodes from two MEAs, 8 microelectrodes per MEA) while the phase was $-74.9 \pm 1.1^{\circ}$. After 60 days, neither the 14 impedance magnitude, $686.7 \pm 98.9 \mathrm{k} \Omega$, nor the phase, $-73.6 \pm 2.8^{\circ}$, were significantly altered, suggesting that novel MEA maintains electrochemical stability for at least 30 days in vitro.

To further assess MEA robustness and tolerance for handling, we examined the stability 17 of impedance characteristics after electropolymerization of microelectrode sites with the conducting polymer poly(3,4-ethylenedioxythiophene) (PEDOT) as described for in vivo 19 microelectrodes [26]. PEDOT deposited with the counterion tetrafluoroborate has been shown to produce a robust, durable, and stable electrochemical interface for microelectrodes [26,27]. As

21 shown in Fig. 2B, coating with PEDOT resulted in a significant alteration in the impedance 22 profile where the initial impedance magnitude and phase at $1 \mathrm{KHz}$ were $6.1 \pm 0.4 \mathrm{k} \Omega$ and $-13.6 \pm$ $230.4^{\circ}$, respectively. After 30 days, the impedance magnitude, $15.3 \pm 1.4 \mathrm{k} \Omega$, and the phase, $-29.5 \pm$ $241.3^{\circ}$ were significantly different from uncoated gold electrodes. 
3.2 Neuronal culture

3

4

2

Following surface preparation, the novel MEA supported cell body adhesion and neuronal growth (Fig 3A). With the maturation of the network, extracellular action potentials could be readily observed across multiple microelectrode recording sites (Fig. 3B); these biphasic waveforms were entirely consistent with extracellular recordings reported previously using conventional MEA platforms [28, 29]. Parallel experiments using MCS MEAs showed virtually identical extracellular potentials (Fig. 3C).

\subsection{Comparative native neuronal activity across MEA platforms}

To examine the suitability of the UT Dallas MEA, we compared the resulting neuronal activity with that derived from the commercially available MCS MEA. As shown in Fig. 4, cortical neuronal networks on each of the MEA platforms after approximately 3 weeks in culture displayed coordinated bursting activity. This observation for both MEA types is entirely consistent with prior work [4,14,30,31]. Multiple experiments were performed to quantitatively and systematically compare the MEA platforms. Electrophysiological parameters such as network mean spiking rate, mean bursting rate, and mean inter-spike interval showed that the activity across the UT Dallas MEAs was statistically indistinguishable from that produced on the MCS MEAs (Table 1). In addition, the activity yield, i.e., the percentage of microelectrode sites that demonstrated resolvable single unit activity, was not different between the MEA types

3.3 Comparative neuronal activity with pharmacological compounds across MEA platforms 
To further probe the consistency of the MEA platforms, we also quantitatively

2 characterized the effects of pharmacological agents on cultured cortical networks. Excitatory

3 reagents, pilocarpine and bicuculline, an inhibitory reagent, memantine, and a neutral

4 biomolecule, $\mathrm{A} \beta_{1-42}$ monomer [32], were applied to networks cultured on both substrate type.

5 Exposure to pilocarpine and bicuculline resulted in an elevation in mean bursting and spiking

6 rate (Fig. $5 \& 6$ ) whereas memantine produced a reduction in mean spiking rate and the $A \beta_{1-42}$

7 monomer failed to markedly alter spike activity. Fig. 6 summarizes the normalized changes in

8 mean spiking rate in response to these compounds for the networks cultured on both MEAs.

9 Linear regression fitting yielded a slope \pm standard error of $0.91 \pm 0.11$, which is statistically

10 indistinguishable from unity. Therefore, the degrees of activity modulation produced by all the

11 reagents were virtually identical for both of the MEA types.

$13 \quad 3.4$ Advantages of the UT Dallas MEA

The use of MEAs for in vitro applications has been well-established since the initial

15 demonstration over 30 years ago [30,33,34]. With more widespread use through commercial

16 availability, a limitation has emerged with respect to the expense largely attributable to the

17 materials, processes, and cleanroom facilities necessary for reproducible MEA fabrication.

18 Unlike virtually all cell culture dishes and chambers, many of the available MEAs are expected

19 to be re-used where specialized and experienced handling is required. In contrast, by leveraging

20 flexible display fabrication approaches, the process for fabricating UT Dallas MEAs relies on

21 materials common to biomedical applications, such as polycarbonate and parylene C, and can be

22 readily scaled in a cost efficient manner. Based on flexible display fabrication costs, we estimate 
1 production costs at nominal volumes to be approximately $\$ 3$ per $\mathrm{cm}^{2}$ such that the novel MEA

2 could be fabricated for as little as $\$ 25$.

3

\section{4. Conclusion}

We have demonstrated a new design for an inexpensive plastic MEA which is readily

6 manufactured and disposable. Comparative analyses of electrophysiological parameters from

7 cortical neuronal network activity from this novel MEA showed virtually identical results with a

8 corresponding commercially available MEA of similar dimensions. We also showed, for the first

9 time, that the primary neuronal cultures on the new disposable MEAs, when compared to networks on

10 these commercially available MEAs, respond similarly to representative excitatory, inhibitory and neutral

11 compounds. Although there have been prior reports on developing novel microelectrode technology,

12 demonstrating the pharmacological consistency is often absent $[15,35]$. Such a finding is not trivial

13 considering that the substrate material could greatly impact the growth and development of primary

14 neuronal cultures [36, 37]. The functional comparison shown in the present study demonstrates that these

15 new disposable MEAs are entirely suitable for in vitro assay applications.

\section{Acknowledgement}

The authors acknowledge support from NSF PFI grant number IIP-1114211 (PI: BE

20 Gnade) and from the DARPA Young Faculty Award Program (PI: WE Voit). The authors would

21 also like to thank Wallace Martin, Jim Burris and Gordon Pollack of the University of Texas at

22 Dallas clean room for many helpful processing suggestions.

\section{References}


[1] A.F.M. Johnstone, G.W. Gross, D.G. Weiss, O.H.-U. Schroeder, A. Gramowski, T.J. Shafer, Microelectrode arrays: a physiologically based neurotoxicity testing platform for the 21st century, Neurotoxicology. 31 (2010) 331-350. doi:10.1016/j.neuro.2010.04.001.

[2] E.W. Keefer, A. Gramowski, D.A. Stenger, J.J. Pancrazio, G.W. Gross, Characterization of acute neurotoxic effects of trimethylolpropane phosphate via neuronal network biosensors, Biosens. Bioelectron. 16 (2001) 513-525.

[3] S.I. Morefield, E.W. Keefer, K.D. Chapman, G.W. Gross, Drug evaluations using neuronal networks cultured on microelectrode arrays, Biosens. Bioelectron. 15 (2000) 383-396.

[4] G. Xiang, L. Pan, L. Huang, Z. Yu, X. Song, J. Cheng, et al., Microelectrode array-based system for neuropharmacological applications with cortical neurons cultured in vitro, Biosens. Bioelectron. 22 (2007) 2478-2484. doi:10.1016/j.bios.2006.09.026.

[5] H. Charkhkar, C. Frewin, M. Nezafati, G.L. Knaack, N. Peixoto, S.E. Saddow, et al., Use of cortical neuronal networks for in vitro material biocompatibility testing, Biosens. Bioelectron. 53 (2014) 316-323. doi:10.1016/j.bios.2013.10.002.

[6] D.J. Bakkum, Z.C. Chao, S.M. Potter, Long-Term Activity-Dependent Plasticity of Action Potential Propagation Delay and Amplitude in Cortical Networks, PLoS ONE. 3 (2008) e2088. doi:10.1371/journal.pone.0002088.

[7] F. Hamilton, T. Berry, N. Peixoto, T. Sauer, Real-time tracking of neuronal network structure using data assimilation, Phys. Rev. E. 88 (2013) 052715. doi:10.1103/PhysRevE.88.052715.

[8] M. Chiappalone, P. Massobrio, S. Martinoia. Network plasticity in cortical assemblies, European Journal of Neuroscience 28 (2008) 221-237.

[9] D. Poli, V.P. Pastore, P. Massobrio. Functional connectivity in in vitro neuronal assemblies, Frontiers in neural circuits 9 (2015).

[10] D.A. Wagenaar, R. Madhavan, J. Pine, S.M. Potter, Controlling Bursting in Cortical Cultures with Closed-Loop Multi-Electrode Stimulation, J. Neurosci. 25 (2005) 680-688. doi:10.1523/JNEUROSCI.4209-04.2005.

[11] A.N. Ide, A. Andruska, M. Boehler, B.C. Wheeler, G.J. Brewer. Chronic network stimulation enhances evoked action potentials, Journal of neural engineering 7 (2010) 016008 .

[12] P. Massobrio, V. Pasquale, S. Martinoia. Self-organized criticality in cortical assemblies occurs in concurrent scale-free and small-world networks, Scientific reports 5 (2015).

[13] C. Tetzlaff, S. Okujeni, U. Egert, F. Worgotter, M. Butz. Self-organized criticality in developing neuronal networks, PLoS Comput. Biol 6 (2010) e1001013.

[14] A. Novellino, B. Scelfo, T. Palosaari, A. Price, T. Sobanski, T.J. Shafer, et al., Development of micro-electrode array based tests for neurotoxicity: assessment of interlaboratory reproducibility with neuroactive chemicals, Front. Neuroengineering. 4 (2011) 4. doi:10.3389/fneng.2011.00004. 
[15] F. Heer, S. Hafizovic, W. Franks, A. Blau, C. Ziegler, A. Hierlemann, CMOS microelectrode array for bidirectional interaction with neuronal networks, IEEE J. SolidState Circuits. 41 (2006) 1620-1629. doi:10.1109/JSSC.2006.873677.

[16] F. Heer, W. Franks, A. Blau, S. Taschini, C. Ziegler, A. Hierlemann, et al., CMOS microelectrode array for the monitoring of electrogenic cells, Biosens. Bioelectron. 20 (2004) 358-366. doi:10.1016/j.bios.2004.02.006.

[17] T. Tateno, J. Nishikawa, A CMOS IC-based multisite measuring system for stimulation and recording in neural preparations in vitro, Front. Neuroengineering. 7 (2014). doi:10.3389/fneng.2014.00039.

[18] J. Müller, M. Ballini, P. Livi, Y. Chen, M. Radivojevic, A. Shadmani, et al., Highresolution CMOS MEA platform to study neurons at subcellular, cellular, and network levels, Lab Chip. 15 (2015) 2767-2780. doi:10.1039/C5LC00133A.

[19] H. Charkhkar, G.L. Knaack, B.E. Gnade, E.W. Keefer, J.J. Pancrazio, Development and demonstration of a disposable low-cost microelectrode array for cultured neuronal network recording, Sens. Actuators B Chem. 161 (2012) 655-660. doi:10.1016/j.snb.2011.10.086.

[20] E.M. Schmidt, J.S. Mcintosh, M.J. Bak, Long-term implants of Parylene-C coated microelectrodes, Med. Biol. Eng. Comput. 26 (1988) 96-101. doi:10.1007/BF02441836.

[21] S. Takeuchi, D. Ziegler, Y. Yoshida, K. Mabuchi, T. Suzuki, Parylene flexible neural probes integrated with microfluidic channels, Lab. Chip. 5 (2005) 519-523. doi:10.1039/b417497f.

[22] S. Kim, R. Bhandari, M. Klein, S. Negi, L. Rieth, P. Tathireddy, et al., Integrated wireless neural interface based on the Utah electrode array, Biomed. Microdevices. 11 (2009) $453-$ 466. doi:10.1007/s10544-008-9251-y.

[23] G.L. Knaack, H. Charkhkar, F.W. Hamilton, N. Peixoto, T.J. O’Shaughnessy, J.J. Pancrazio, Differential responses to $\omega$-agatoxin IVA in murine frontal cortex and spinal cord derived neuronal networks, Neurotoxicology. 37 (2013) 19-25. doi:10.1016/j.neuro.2013.03.002.

[24] T.J. Shafer, S.O. Rijal, G.W. Gross, Complete inhibition of spontaneous activity in neuronal networks in vitro by deltamethrin and permethrin, Neurotoxicology. 29 (2008) 203-212. doi:10.1016/j.neuro.2008.01.002.

[25] D.A. Robinson, The electrical properties of metal microelectrodes, Proc. IEEE. 56 (1968) 1065-1071. doi:10.1109/PROC.1968.6458.

[26] H.S. Mandal, G.L. Knaack, H. Charkhkar, D.G. McHail, J.S. Kastee, T.C. Dumas, et al., Improving the performance of poly(3,4-ethylenedioxythiophene) for brain-machine interface applications, Acta Biomater. 10 (2014) 2446-2454. doi:10.1016/j.actbio.2014.02.029.

[27] H.S. Mandal, J.S. Kastee, D.G. McHail, J.F. Rubinson, J.J. Pancrazio, T.C. Dumas, Improved Poly(3,4-Ethylenedioxythiophene) (PEDOT) for Neural Stimulation, Neuromodulation J. Int. Neuromodulation Soc. (2015). doi:10.1111/ner.12285.

[28] Y. Nam, B.C. Wheeler, In vitro microelectrode array technology and neural recordings, Crit. Rev. Biomed. Eng. 39 (2011) 45-61.

[29] M. Chiappalone, A. Vato, M.B. Tedesco, M. Marcoli, F. Davide, S. Martinoia, Networks of neurons coupled to microelectrode arrays: a neuronal sensory system for pharmacological applications, Biosens. Bioelectron. 18 (2003) 627-634. 
[30] G.W. Gross, W.Y. Wen, J.W. Lin, Transparent indium-tin oxide electrode patterns for extracellular, multisite recording in neuronal cultures, J. Neurosci. Methods. 15 (1985) 243-252. doi:10.1016/0165-0270(85)90105-0.

[31] Y. Jimbo, A. Kawana, P. Parodi, V. Torre, The dynamics of a neuronal culture of dissociated cortical neurons of neonatal rats, Biol. Cybern. 83 (2000) 1-20. doi:10.1007/PL00007970.

[32] H. Charkhkar, S. Meyyappan, E. Matveeva, J.R. Moll, D.G. McHail, N. Peixoto, R.O. Cliff, J.J. Pancrazio. Amyloid beta modulation of neuronal network activity in vitro, Brain research 1629 (2015) 1-9.

[33] G.W. Gross, E. Rieske, G.W. Kreutzberg, A. Meyer, A new fixed-array multimicroelectrode system designed for long-term monitoring of extracellular single unit neuronal activity in vitro, Neurosci. Lett. 6 (1977) 101-105.

[34] G.W. Gross, Simultaneous Single Unit Recording in vitro with a Photoetched Laser Deinsulated Gold Multimicroelectrode Surface, IEEE Trans. Biomed. Eng. BME-26 (1979) 273-279. doi:10.1109/TBME.1979.326402.

[35] N.C. Hogan, G. Talei-Franzesi, O. Abudayyeh, A. Taberner, I. Hunter. Low-cost, flexible polymer arrays for long-term neuronal culture. Engineering in Medicine and Biology Society (EMBC), 2012 Annual International Conference of the IEEE (2012) 803-806.

[36] S.C. Bayliss, L.D. Buckberry, I. Fletcher, M.J. Tobin. The culture of neurons on silicon, Sensors and Actuators A: Physical 74 (1999) 139-142

[37] L.A. Flanagan, Y.-E. Ju, B. Marg, M. Osterfield, P.A. Janmey. Neurite branching on deformable substrates, Neuroreport 13 (2002) 2411

9

0

1

2

33


Fig. 3. Cultured neuronal network on a UT Dallas MEA substrate (A). Representative activity from a single microelectrode recording site from a UT Dallas MEA showing two distinct units (B). Similar activity from a sister neuronal network culture on a MCS MEA (C).

Fig. 4. Raster plots of extracellular activity from cortical neuronal networks cultured for at least 21 days in vitro on a representative UT Dallas MEA (A) and a typical MCS MEA (B).

Fig. 5. Raster plots of extracellular activity from cortical neuronal networks exposed to $1 \mathrm{mM}$ pilocarpine on UT Dallas (A) and MCS (B) MEAs. Neuronal networks on both substrates

27 responded similarly to the reagent.

Fig. 6. Normalized spiking rates for neuronal networks on UT Dallas vs. MCS MEAs in response to $1 \mathrm{mM}$ pilocarpine (o), $5 \mu \mathrm{M}$ bicuculline ( $\square)$, $5 \mu \mathrm{M} A \beta_{1-42}$ monomer $(\diamond)$, and $20 \mu \mathrm{M}$ memantine 
1 Table 1. Comparison of neuronal network parameters from sister cortical cultures seeded on

2 novel MEAs and MCS MEAs. None of the parameters were statistically different between the 3 MEA platforms.

4 
Table 1.

\begin{tabular}{lccccc}
\hline & $\begin{array}{c}\text { Network } \\
\text { Number }\end{array}$ & Spike rate (Hz) & $\begin{array}{c}\text { Burst rate } \\
\left(\mathbf{m i n}^{-\mathbf{1}}\right)\end{array}$ & $\begin{array}{c}\text { Inter-spike } \\
\text { interval (s) }\end{array}$ & Yield (\%) \\
\hline UT Dallas MEAs & 11 & $2.8 \pm 0.3$ & $4.8 \pm 1.0$ & $1.8 \pm 0.1$ & $67 \pm 6$ \\
\hline MCS MEAs & 9 & $2.5 \pm 0.5$ & $4.3 \pm 0.8$ & $1.7 \pm 0.2$ & $74 \pm 11$ \\
\hline
\end{tabular}



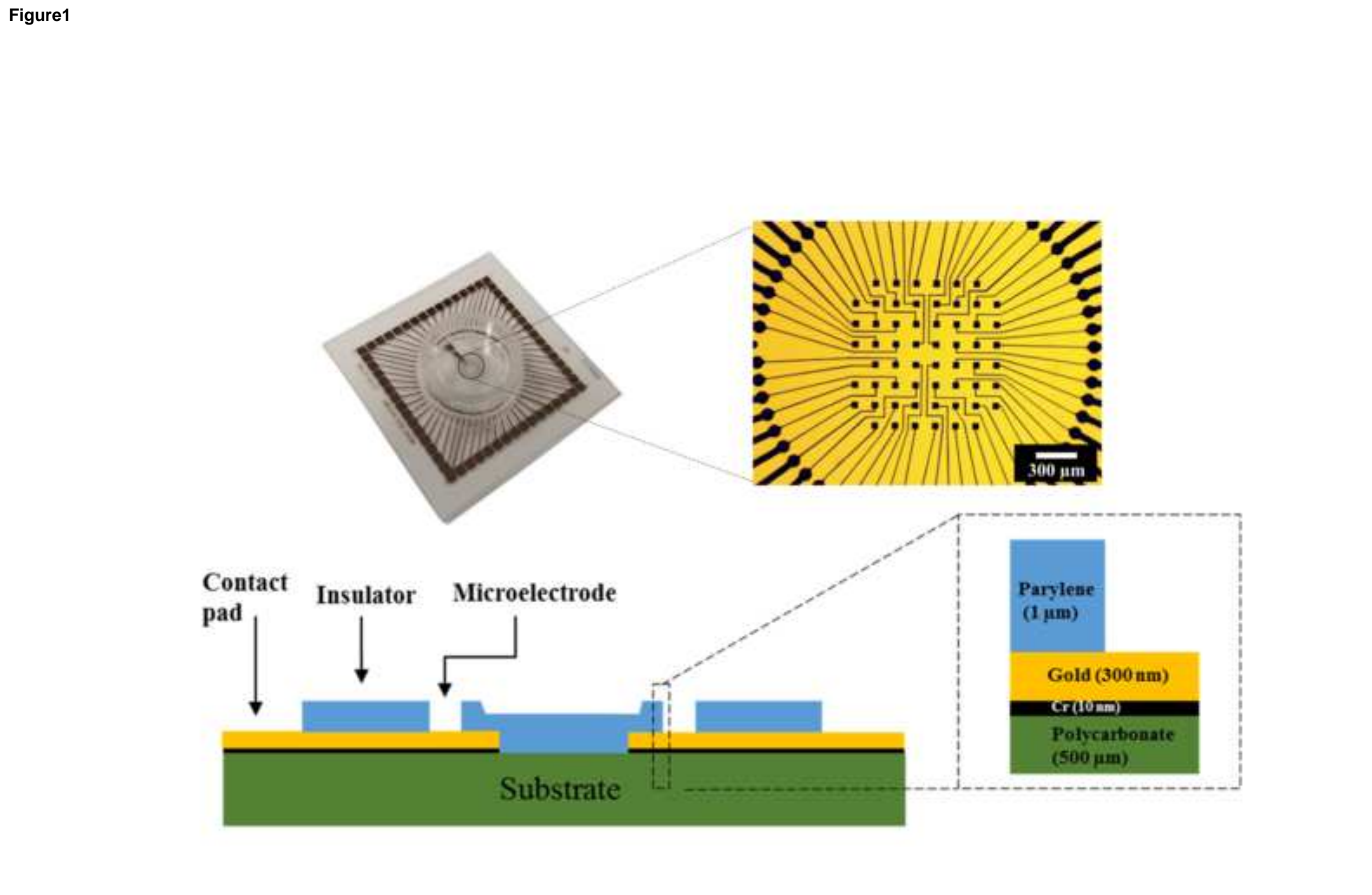
A
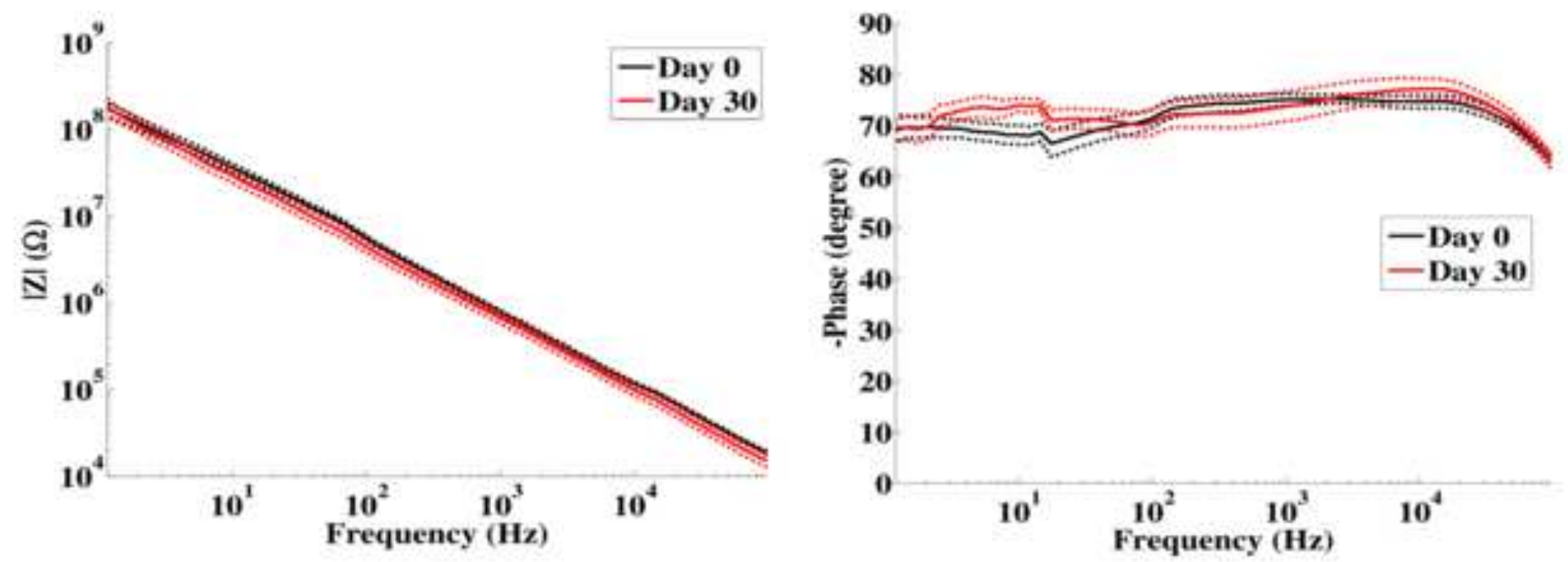

B
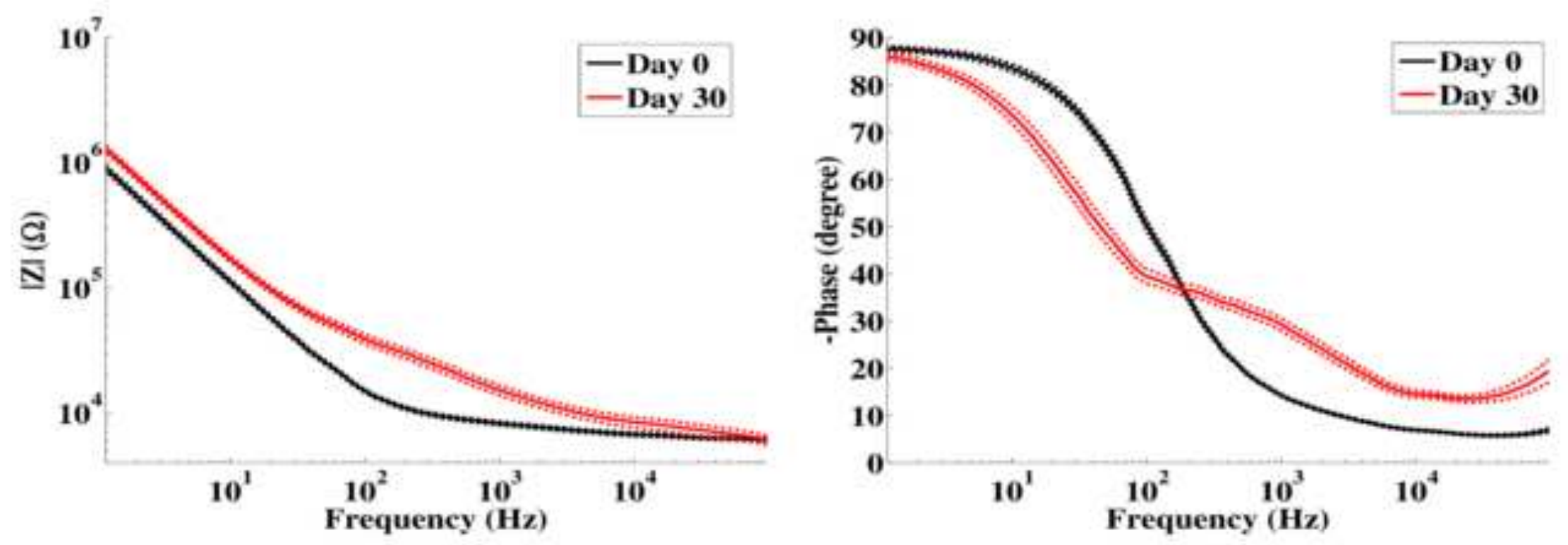
C

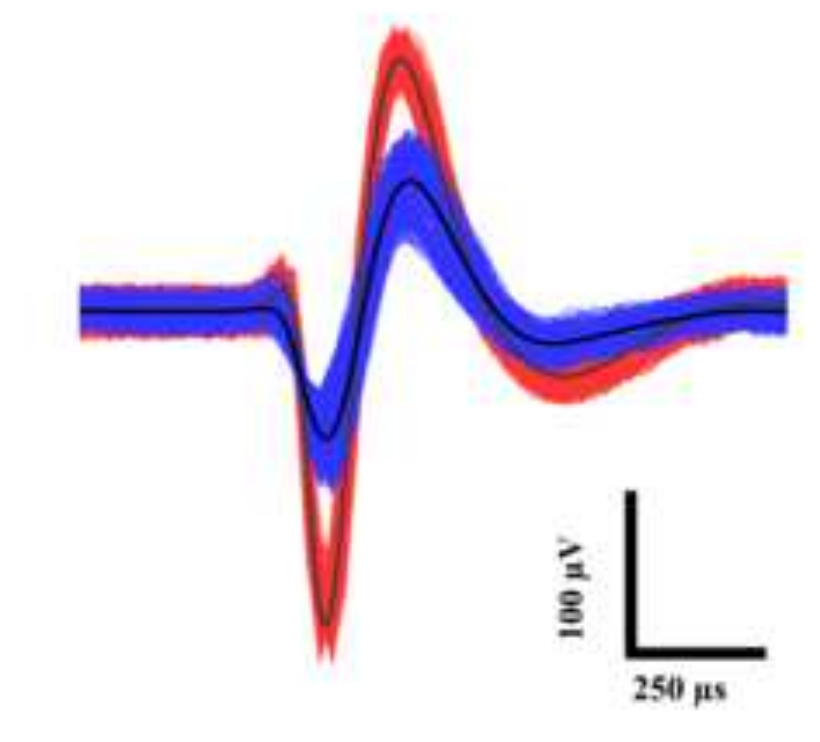

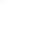

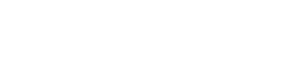

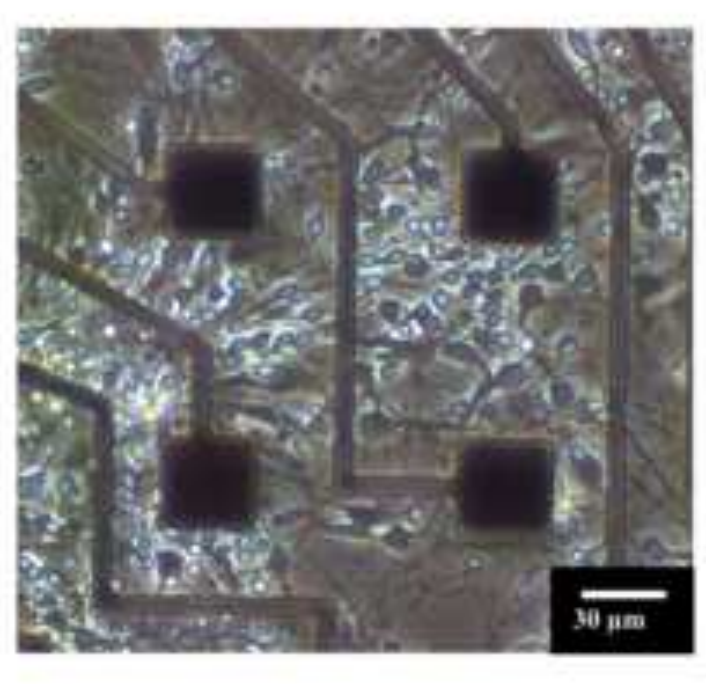

A
B

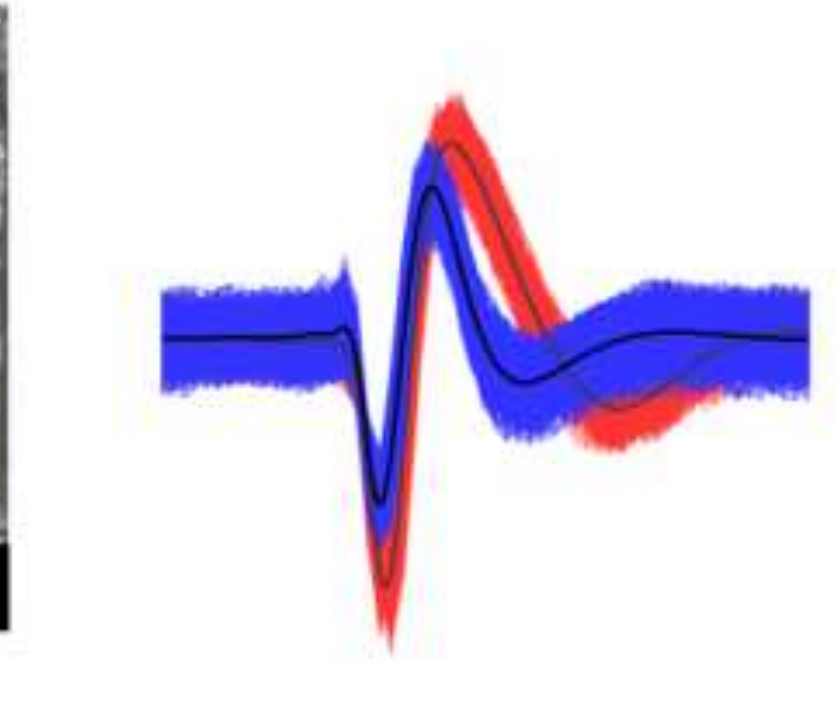


A

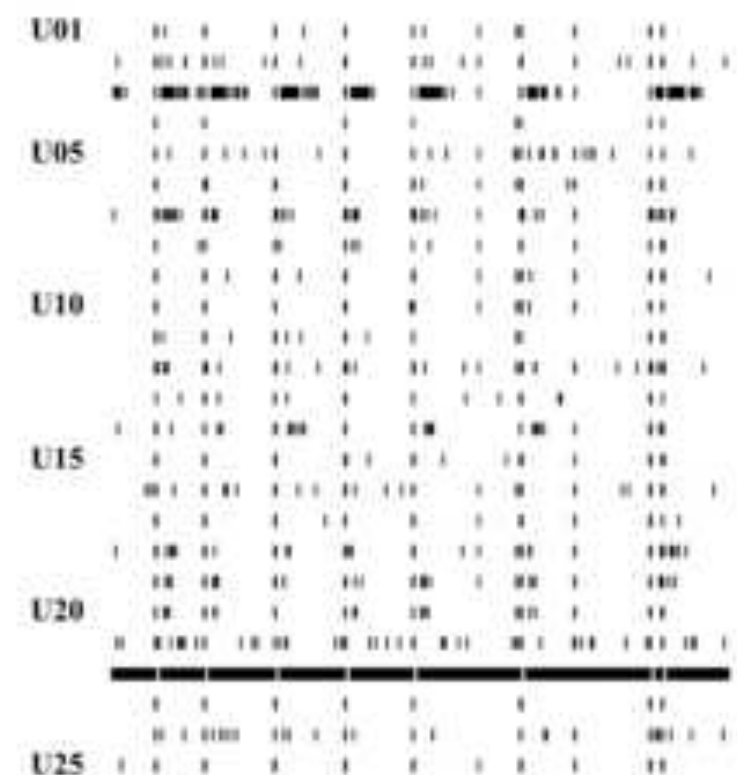

B

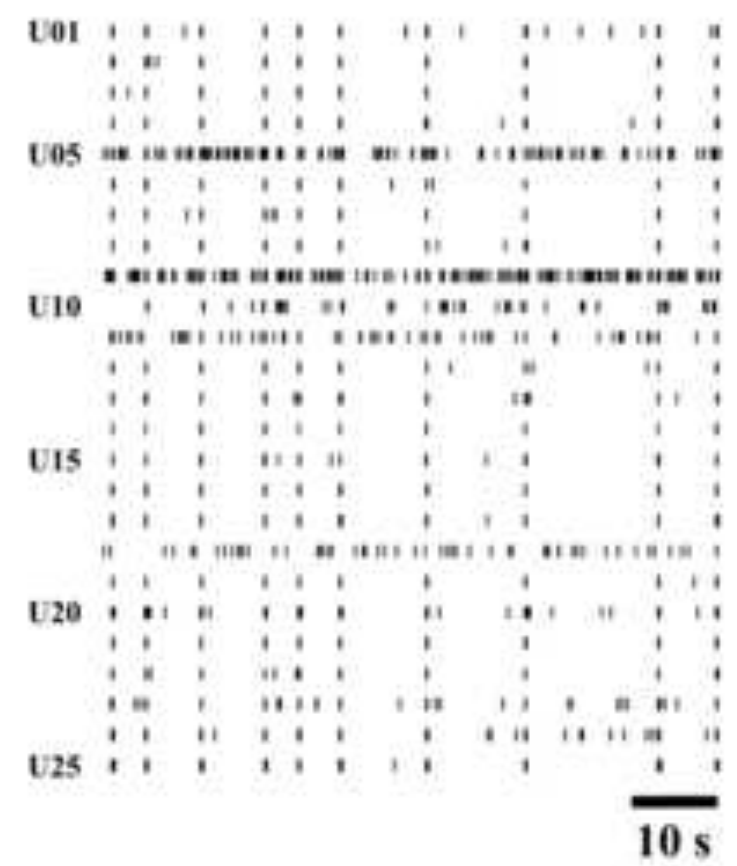


Baseline

tat

\begin{tabular}{|c|c|c|c|c|c|c|c|c|c|}
\hline 贯! & III & 1 & (1) & & $=$ & E & $=$ & & a \\
\hline 1 & 1 & I & 1 & 1 & 1 & III & " & & II \\
\hline 1 & 11 & 1 & 11 & & 11 & 1 & $t$ & 1 & 11 \\
\hline 1 & 1 & 1 & 1 & & 11 & 11 & 111 & & 1 \\
\hline 111 & 1 & III & 1111 & & It & II & I $\mathrm{E}$ & & 1 \\
\hline it & 1 & 1 & 1 & & 11 & 111 & 11 & & I \\
\hline 1 & 1 & 11 & II) & 1 & 111 & 11 & 10 & & 11 \\
\hline I & 1 & 11 & 1 & & 1 & 1 & 1 & & I \\
\hline II & 11 & $\mathrm{I}$ & II & & 1 & 1 & 111 & & 111 \\
\hline
\end{tabular}

v16

MCS
(⿻)

Les
$\begin{array}{llllllllll}1 & 1 & 1 & 1 & 1 & 1 & 1 & 11 & 1\end{array}$

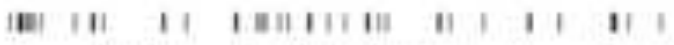

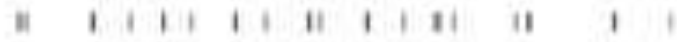

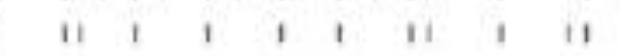

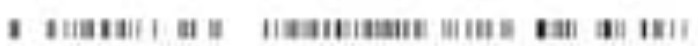

$1+1+1111 \quad 1 \quad 11$

$11101111,1,11$

$\begin{array}{lllllllllll}1 & 11 & 1 & 1 & 1 & 1 & 11 & 1 & 1 & 1\end{array}$

$1,1,1,111$

tง

\section{$1 \mathrm{~m}$ M Pilocarpine}

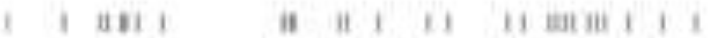

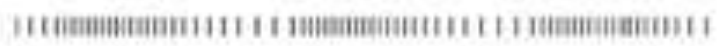

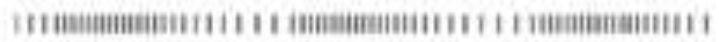

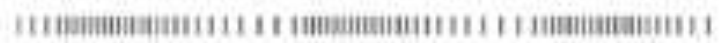

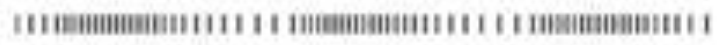

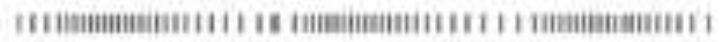

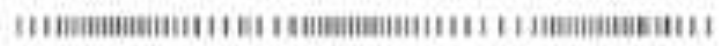

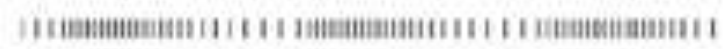

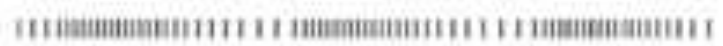

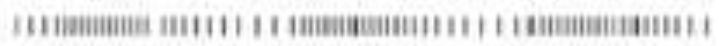

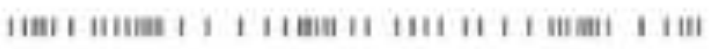

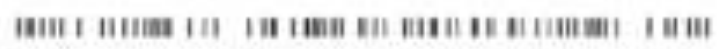
I I

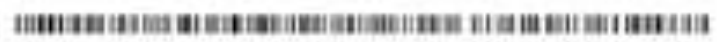

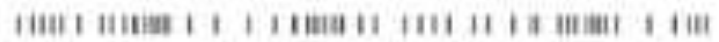

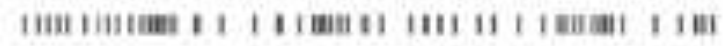

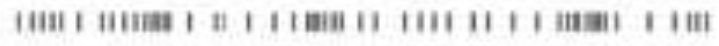
t 1 \& G

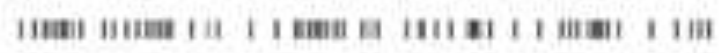




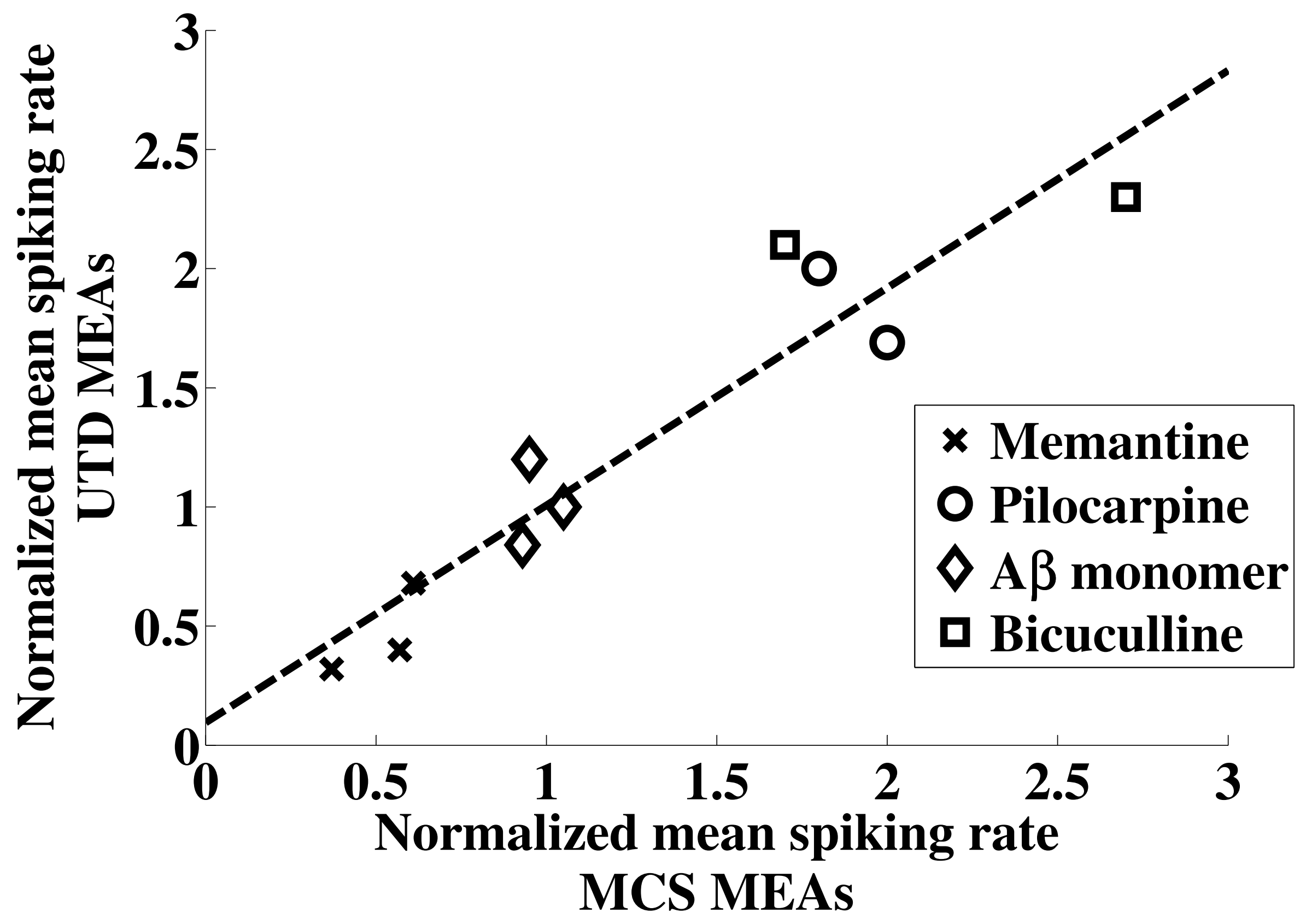

